\title{
Usage of glucometer is associated with improved glycaemic control in type 2 diabetes mellitus patients in Malaysian public primary care clinics: an open-label, randomised controlled trial
}

\author{
Mastura Ismail ${ }^{1}$, MMed, Chong-Lieng $\underline{\text { Teng}}{ }^{2}$, MMed, Mimi Omar ${ }^{3}$, MMed, Bee Kiau $\underline{\mathrm{Ho}}^{4}$, Mmed,
} Zainab Kusiar ${ }^{5}$, mMed, Ruziaton Hasim $^{6}$, mMed

\begin{abstract}
INTRODUCTION Self-monitoring of blood glucose (SMBG) has been underutilised. We conducted an open-label, randomised controlled trial to assess the feasibility of introducing SMBG in primary care clinics in Malaysia.

METHODS This was an open-label, randomised controlled trial conducted in five public primary care clinics in Malaysia. Patients with type 2 diabetes mellitus (age range 35-65 years) not performing SMBG at the time of the study were randomised to receive either a glucometer for SMBG or usual care. Both groups of patients received similar diabetes care from the clinics.

RESULTS A total of 105 patients with type 2 diabetes mellitus were enrolled. Of these, 58 and 47 were randomised to intervention and control groups, respectively. After six months, the glycated haemoglobin (HbA1c) level in the intervention group showed a statistically significant improvement of $1.3 \%(p=0.001 ; 95 \%$ confidence interval $0.6-2.0$ ), relative to the control group that underwent usual care. The percentages of patients that reached the $\mathrm{HbA1c}$ treatment target of $\leq 7 \%$ were $14.0 \%$ and $32.1 \%$ in the control and intervention groups $(p=0.036)$, respectively. CONCLUSION The usage of a glucometer improved glycaemic control, possibly due to the encouragement of greater self-care in the intervention group.
\end{abstract}

Keywords: diabetes mellitus, Malaysia, primary care, randomised controlled trial, self-monitoring of blood glucose

\section{INTRODUCTION}

National population surveys have highlighted the increasing prevalence of diabetes mellitus (DM) in Malaysia, with the prevalence reaching $14.9 \%$ in 2006. . $^{(1)}$ The data in a national DM audit showed that only $18.1 \%$ of patients with DM achieved a glycated haemoglobin (HbA1c) level of $<6.5 \%$. $^{(2)}$ Similarly, several other studies also showed poor glycaemic control in Malaysia. ${ }^{(3,4)}$ Poor diabetes control leads to macroand microangiopathy, while good glycaemic control can improve the outcome in types 1 and 2 DM..$^{(5,6)}$ All diabetes care strategies should aim to achieve strict glycaemic control. As a tool for attaining target glycaemic control, self-monitoring of blood glucose (SMBG) has become a common practice in developed countries. ${ }^{(6,7)}$ However, in low-income countries, it is not entirely clear whether SMBG can produce the level of glycaemic control seen in randomised controlled trials conducted in developed countries. ${ }^{(8)}$ This is especially pertinent in view of patients' lower levels of education and income, which impact Malaysia's public healthcare system. As there are still relatively few controlled studies on the efficacy of SMBG in developing countries, ${ }^{(9-11)}$ we aimed to add further data in this area by conducting a randomised controlled study in the Malaysian public primary care setting. The main objective of this study was to determine the difference in diabetes control between patients who undertake SMBG and those who have their blood glucose level monitored in public health clinics.

\section{METHODS}

This was an open-label, randomised controlled trial conducted on patients with type 2 DM (aged 35-65 years) who required either an oral antidiabetic agent or insulin, and who had $\mathrm{HbA} 1 \mathrm{c}>6.5 \%$. The criteria for exclusion from the study were: (a) existing SMBG; (b) pregnancy; (c) acute infection; (d) diabetic retinopathy; (e) overt proteinuria confirmed by dipstick; and (f) serum creatinine $>176.8 \mu \mathrm{mol} / \mathrm{L}$.

The study was conducted at two health clinics located in Negeri Sembilan, and at three others in Selangor. These clinics are typical of the many public community health centres in Malaysia, which provide comprehensive and continuing medical care to the population. Each clinic tasked a nurse to recruit 30 patients according to the study's inclusion and exclusion criteria. The recruited patients voluntarily consented to join the study and made an appointment for the baseline study visit. The study's

\footnotetext{
${ }^{1}$ Seremban 2 Health Clinic, ${ }^{2}$ Department of Family Medicine, International Medical University, Seremban, Negeri Sembilan, ${ }^{3}$ Kelana Jaya Health Clinic, ${ }^{4}$ Shah Alam Health Clinic, Selangor, ${ }^{5}$ Nilai Health Clinic, Nilai, Negeri Sembilan, ${ }^{6}$ Teluk Datok Health Clinic, Selangor, Malaysia

Correspondence: Dr Mastura Ismail, Family Medicine Specialist, Klinik Kesihatan Seremban 2, Jalan S2 A2, Seremban 2, 70300 Seremban, Negeri Sembilan Darul Khusus, Malaysia.drmi68@yahoo.com
} 
sampling frame comprised DM patients who attended these five clinics during the three-month recruitment phase from April to June, 2007. During the baseline study visit at each clinic, the nurse assigned a consecutive number, ranging from 1 to 10, to each recruited patient. The numbers were used to randomly assign patients to different groups. Patients 3, 4, 7, 9 and 10 were assigned to Group 1 (control group; i.e. patients whose blood glucose level will only be monitored in public health clinics), while Patients 1, 2, 5, 6 and 8 were assigned to Group 2 (intervention group; i.e. patients who will undertake SMBG at home). Patients in both groups were each allocated two monthly clinic visits with doctors, but patients in Group 2 were additionally allocated monthly appointments with the nurses to record their SMBG levels. Thus, a total of 150 patients made appointments for the baseline study visit. All patients were treated and followed up by family medicine specialists and medical officers, with the aim of achieving fasting plasma glucose $<6.0 \mathrm{mmol} / \mathrm{L}$ and $\mathrm{HbA} 1 \mathrm{c}<6.5 \%$. Neither the patients nor the doctors were blinded. Patient records were tagged to indicate their assigned group.

All patients (Groups 1 and 2) received similar health education, as recommended in the Malaysian Clinical Practice Guidelines $^{(12)}$ (CPG) on the management of DM, which highlights the need for strict glycaemic control, diet control, blood glucose monitoring, and knowledge on how to adjust the dose of oral hypoglycaemic agents (OHA) or insulin, as well as treatment of hypoglycaemia. In addition to the health education session, patients in Group 2 were offered two-day classes that included practical demonstrations of SMBG, during which the usage of the glucometer was explained. Patients were supplied a glucometer (OneTouch ${ }^{\circledR}$ Ultra ${ }^{\circledR}$; Johnson \& Johnson, CA, USA) with reagent test strips (OneTouch ${ }^{\circledR}$ Ultra $^{\circledR}$; Johnson \& Johnson, CA, USA) at no charge, after they demonstrated the skill needed to use the device.

All patients in Group 2 were advised to monitor their blood glucose levels (either during fasting, two hours after breakfast, or two hours after meals) and to keep a record in their logbooks. The frequency of SMBG depends on the level of diabetes control required, according to the guidelines set in the Malaysian CPG on the management of diabetes. If the test result was found to be above the set target value (i.e. fasting blood glucose $>6.0 \mathrm{mmol} / \mathrm{L}$; postprandial blood glucose $>7.8 \mathrm{mmol} / \mathrm{L}$ ), the patient was advised to adjust the dose of $\mathrm{OHA} /$ insulin accordingly and recheck the blood glucose level of that particular time (either during fasting or postprandial), after four to five days. Patients in Group 1 were asked to visit the doctor at intervals of two months, and antidiabetic treatments were modified if needed. They were also free to report at any time should they face any difficulty. Group 2 patients were also required to visit their doctor at intervals of

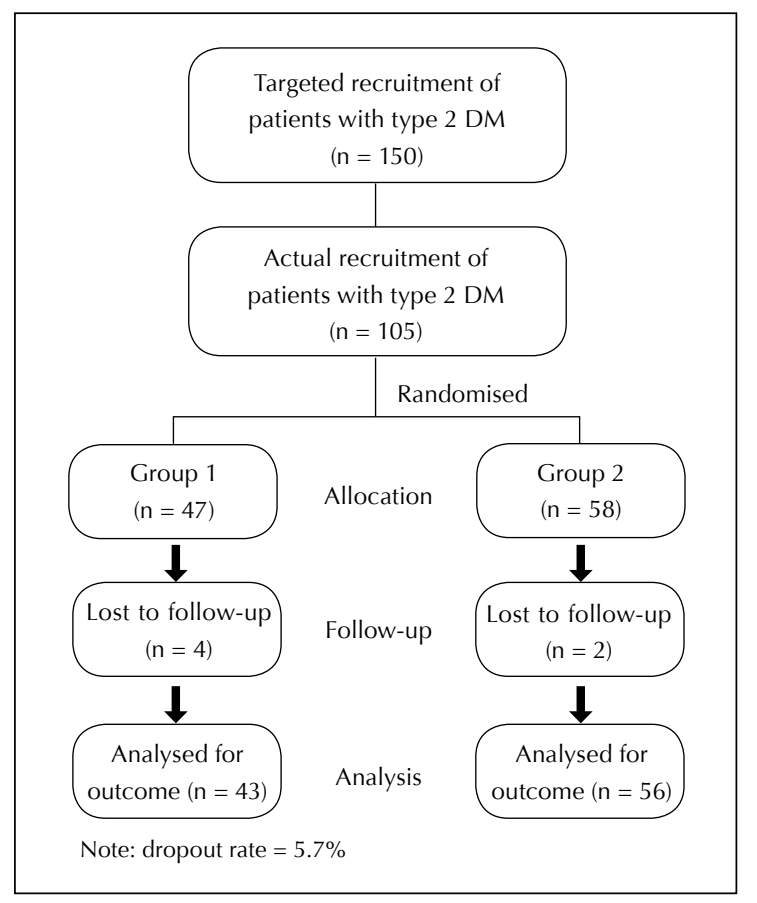

Fig. 1 Flow chart demonstrates subject recruitment, follow-up and outcome. DM: diabetes mellitus; SMGB: self-monitoring of blood glucose

two months. However, in addition to that, Group 2 patients were also required to see the nurse every month to record their SMBG results.

For both groups, tests for either fasting blood glucose or two-hour postprandial blood glucose were done at each visit, and $\mathrm{HbA1c}$ tests were ordered every three to six months. Tests for fasting cholesterol, triglycerides and serum creatinine levels were performed every six months. Weight was taken with the patient barefoot and in light clothing, and blood pressure was recorded in the sitting position using a standard mercury sphygmomanometer. The duration of the study period was six months.

Sample size was calculated based on the method described by Campbell et al. ${ }^{(13)}$ In order to detect a difference of $1 \%$ in patients' $\mathrm{HbA} 1 \mathrm{c}$ levels at the six month follow-up (standard deviation $=2 \%, \alpha=5 \%, \beta=20 \%$ ), 64 patients were needed in each arm. However, assuming a $20 \%$ dropout rate, we needed a total of 150 patients (75 in each arm). Each centre thus recruited 15 patients for each of the intervention and control arms.

This study was approved by the Research and Ethics Committee, International Medical University, Malaysia, and written informed consent was obtained from all patients involved in this study prior to data collection. According to protocol, data analysis was performed using the Statistical Package for the Social Sciences Windows version 16.0 (SPSS Inc, Chicago, IL, USA). Categorical variables were compared using chi-square test, and continuous variables were compared using t-test or analysis of variance, as appropriate. The level of statistical significance was set at $\mathrm{p}<0.05$. 
Table I. Comparison between Groups 1 and 2.

\begin{tabular}{|c|c|c|c|}
\hline Characteristic & $\begin{array}{l}\text { Group } 1 \\
(n=47)\end{array}$ & $\begin{array}{l}\text { Group } 2 \\
(n=58)\end{array}$ & p-value \\
\hline Mean age \pm SD (yrs) & $52.7 \pm 7.9$ & $54.0 \pm 9.7$ & 0.485 \\
\hline $\begin{array}{l}\text { Ethnicity (no. [\%]) } \\
\text { Malays } \\
\text { Chinese } \\
\text { Indians }\end{array}$ & $\begin{array}{r}29(61.7) \\
12(25.5) \\
6(12.8)\end{array}$ & $\begin{aligned} 35 & (60.3) \\
7 & (12.1) \\
16 & (27.6)\end{aligned}$ & 0.070 \\
\hline Married (no. [\%]) & $45(95.7)$ & $53(91.4)$ & 0.470 \\
\hline $\begin{array}{l}\text { Education level (no. [\%]) } \\
\text { No formal education or primary education only } \\
\text { Secondary or tertiary education }\end{array}$ & $\begin{array}{l}27(57.4) \\
20(42.6)\end{array}$ & $\begin{array}{l}23(39.7) \\
35(60.3)\end{array}$ & 0.167 \\
\hline $\begin{array}{l}\text { Total household income/mth (no. [\%]) } \\
\text { < RM 1,000 } \\
\text { RM 1,001-3,000 } \\
>\text { RM 3,001 }\end{array}$ & $\begin{array}{r}20(42.6) \\
22(46.8) \\
5(10.6)\end{array}$ & $\begin{array}{r}15(25.9) \\
37(63.8) \\
6(10.3)\end{array}$ & 0.086 \\
\hline Mean duration of $\mathrm{DM} \pm S \mathrm{D}^{*}$ (mths) & $72.5 \pm 53.3$ & $76.0 \pm 71.1$ & 0.776 \\
\hline Current/ex-smoker (no. [\%]) & $6(12.8)$ & $8(13.8)$ & 0.434 \\
\hline Performs physical activity ${ }^{+}$(no. [\%]) & $29(61.7)$ & $43(74.1)$ & 0.172 \\
\hline DM complication present (no. [\%]) & $11(23.4)$ & $9(15.5)$ & 0.346 \\
\hline With history of hospitalisation (no. [\%]) & $1(2.1)$ & $5(8.6)$ & $0.221^{*}$ \\
\hline Educated on DM (no. [\%]) & $38(80.9)$ & $53(91.4)$ & 0.115 \\
\hline Mean systolic $\mathrm{BP} \pm \mathrm{SD}(\mathrm{mmHg})$ & $131.7 \pm 18.4$ & $131.5 \pm 15.2$ & 0.932 \\
\hline Mean diastolic BP (mmHg) & $80.2 \pm 6.9$ & $79.2 \pm 8.4$ & 0.488 \\
\hline Mean waist circumference \pm SD $(\mathrm{cm})$ & $92.2 \pm 9.0$ & $93.6 \pm 9.5$ & 0.447 \\
\hline Mean BMI \pm SD $\left(\mathrm{kg} / \mathrm{m}^{2}\right)$ & $27.7 \pm 4.0$ & $27.2 \pm 4.1$ & 0.529 \\
\hline Mean HbA1c \pm SD (\%) & $8.9 \pm 2.0$ & $9.2 \pm 2.1$ & 0.458 \\
\hline Mean triglyceride level \pm SD $(\mathrm{mmol} / \mathrm{L})$ & $1.9 \pm 1.2$ & $2.3 \pm 1.4$ & 0.174 \\
\hline
\end{tabular}

*The mean duration of DM was obtained from patients' records, or from patients' self-reports, if the information was not recorded. ${ }^{\dagger} \mathrm{Minimum}$ of 30 mins of exercise per session for at least 3 times per week based on self-reporting. ${ }^{~}$ Fisher's exact test.

BMI: body mass index; BP: blood pressure; DM: diabetes mellitus; SD; standard deviation; SMBG: self-monitoring of blood glucose

\section{RESULTS}

This study was conducted from April to December, 2007 in five public health clinics in Malaysia. Out of the 150 patients selected, only 105 patients attended the baseline study visit. Reasons for not participating were: recently purchased own glucometers, lack of interest, unable to come for scheduled visits and logistic factors. The recruited participants were randomised into two groups; 58 patients were supplied glucometers (Group 2) and 47 patients were not supplied glucometers (Group 1). In Group 2, two patients declined to continue the study, while in Group 1, four patients did not complete the study one patient was referred to the hospital for diabetes complication, and three defaulted on their clinic appointments. The recruitment and follow-up process is summarised in Fig. 1. There were no differences observed in the patients' socio-demographic distribution, clinical data, and physical and laboratory findings at baseline study (Table I).

After six months, the Group 2 patients' HbA1c levels showed statistically significant improvement, relative to those Group 1, with a difference of $1.3 \%$ ( $p=0.001 ; 95 \%$ confidence interval $[\mathrm{Cl}]$ 0.6-2.0) favouring SMBG. The absolute mean improvement in the HbA1c levels of Group 2 was $0.9 \%$, as compared to $-0.4 \%$ in Group 1 . The difference $(0.7 \mathrm{mmol} / \mathrm{L})$ in triglyceride levels between Groups 1 and 2 was statistically significant $(p=0.029)$ (Table II).

The average frequency of SMBG testing was 2.8 times per week. At the start of the study, the percentages of DM patients achieving the target $\mathrm{HbA} 1 \mathrm{c}$ level of $\leq 7 \%$ in Groups 1 and $2(17.0 \%$ and $13.8 \%$, respectively) were similar $(p=0.647)$. However, six months later, the percentage of DM patients reaching the treatment target fell to $14.0 \%$ in Group 1 , and increased to $32.1 \%$ in Group 2 (odds ratio 2.9, 95\% Cl 1.04-8.17; $\mathrm{p}=0.036$ ) (Fig. 2).

\section{DISCUSSION}

This study observed that SMBG usage among patients with type $2 \mathrm{DM}$ in five government health clinics in Malaysia significantly improved glycaemic control; after six months, HbA1c levels in the intervention group (i.e. Group 2) lowered by $1.3 \%$, relative to the control group (i.e. Group 1). Similarly, a systematic review ${ }^{(14)}$ of five randomised controlled trials ${ }^{(15-19)}$ 
Table II. Reduction of physical and laboratory findings post intervention as compared to baseline.

\begin{tabular}{lccccc}
\hline Characteristic & $\begin{array}{l}\text { Group 1 } \\
(\mathbf{n}=\mathbf{4 3 )}\end{array}$ & $\begin{array}{l}\text { Group 2 } \\
(\mathbf{n}=\mathbf{5 6})\end{array}$ & $\begin{array}{c}\text { Mean difference } \\
\text { (Group 1 - Group 2) }\end{array}$ & 95\% Cl & p-value \\
\hline Systolic BP (mmHg) & $1.5 \pm 18.4$ & $3.3 \pm 16.5$ & -1.8 & -8.8 to 5.3 & 0.623 \\
Diastolic BP (mmHg) & $1.0 \pm 9.2$ & $1.3 \pm 9.4$ & -0.3 & -4.4 to 3.4 & 0.864 \\
Waist circumference (cm) & $-0.1 \pm 3.5$ & $1.6 \pm 4.8$ & -1.7 & -3.6 to 0.2 & 0.079 \\
BMI $\left(\mathrm{kg} / \mathrm{m}^{2}\right)$ & 0.8 & 1.0 & -0.2 & -0.4 to 0.4 & 0.962 \\
HbA1c $(\%)$ & $-0.4 \pm 1.3$ & $0.9 \pm 2.1$ & -1.3 & -2.0 to -0.6 & $0.001^{*}$ \\
Total cholesterol (mmol/L) & $0.05 \pm 1.4$ & $0.04 \pm 1.0$ & -0.01 & -0.8 to 0.1 & 0.14 \\
Triglyceride (mmol/L) & $-0.5 \pm 1.9$ & $0.2 \pm 1.2$ & -0.7 & -1.3 to 0.1 & $0.029^{*}$ \\
\hline
\end{tabular}

Note: Data is expressed as mean \pm standard deviation, except for data on BMI. *p-value is statistically significant.

BMI: body mass index; BP: blood pressure; $\mathrm{Cl}$ : confident interval; SMBG: self-monitoring of blood glucose

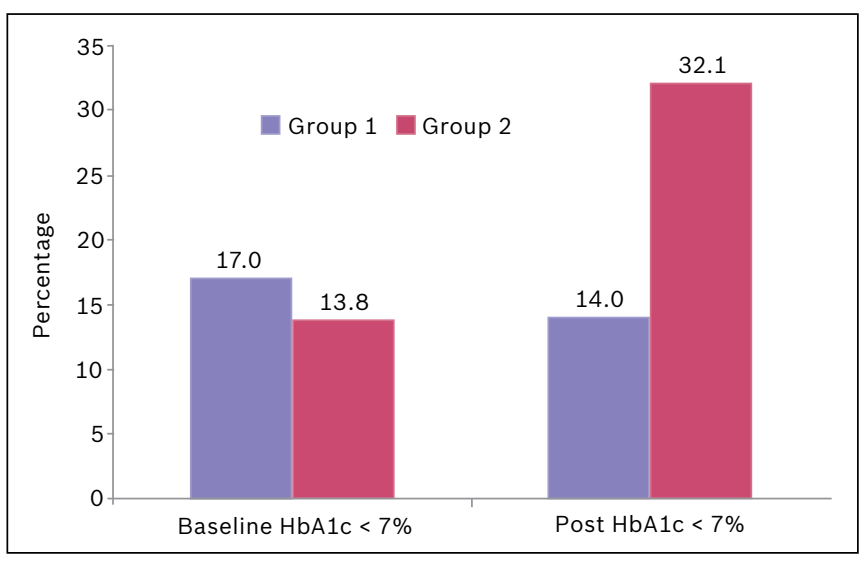

Fig. 2 Bar graph shows the percentages of diabetic patients achieving target $\mathrm{HbA} 1 \mathrm{c}$ level at baseline and at six months post intervention.

patients with on type 2 DM showed that the overall effect was a statistically significant decrease of $0.39 \%$ in the HbA1c levels $(95 \% \mathrm{Cl}-0.56$ to -0.21$)$ of the SMBG group. However, several systematic reviews show that SMBG is only modestly effective in reducing HbA1c. ${ }^{(20-22)}$

In this study, the calculated sample size was 75 patients per group, which was inflated by 1.2 times to take into account an expected dropout rate of $20 \%$. Even though the sample size was not achieved, we managed to find statistical significance because the change in $\mathrm{HbA1c}$ levels was larger than expected (1.3\% instead of $1.0 \%$ ). Also, the actual dropout rate was lower than the expected $20 \%$. This study managed to show that a better reduction of HbA1c levels was achieved in Group 2. This could be due to a higher motivation among Group 2 patients to become active participants in self-care.

Self-monitoring can motivate patients to become active participants in their own care via regular SMBG demonstration of the positive effects of medications, diet and exercise on blood glucose levels. ${ }^{(6)}$ However, a national audit on DM conducted in government health clinics in Malaysia showed that only $10 \%$ of DM patients performed SMBG. ${ }^{(4)}$ Similarly, other local studies also found that about $15 \%$ of DM patients performed SMBG. ${ }^{(23,24)}$ An important step in achieving optimal blood glucose monitoring behaviour is to identify and resolve the barriers to SMBG. ${ }^{(25)}$ The practice of SMBG may increase with the reduction of patients' financial burdens through government subsidies, thus leading to better glycaemic control and reduced diabetic complications. ${ }^{(10,25)}$

It has been predicted that a lifetime incidence of diabetesrelated complications can be reduced if self-monitoring is performed seven or more times per week when compared to the absence of self-monitoring. ${ }^{(26)}$ However, it has also been suggested that less frequent testing of one to two times per week may be more cost-effective in type 2 DM patients who are not on insulin. ${ }^{(27)}$ Other randomised controlled trials ${ }^{(28-30)}$ found no convincing evidence to recommend routine SMBG in reasonably well-controlled, non-insulin-treated type 2 DM patients and those who are newly diagnosed. ${ }^{(31)}$ However, if the $\mathrm{HbA1c}$ level remains above $8 \%$, SMBG may provide motivation for better medication adherence and lifestyle changes. ${ }^{(32)}$ Unlike other studies, ${ }^{(31,33)}$ our study did not find any significant relation to age, gender, ethnicity, education level or financial status.

Although the findings of our study show that SMBG is effective in a trial setting, further studies need to be done to evaluate its effectiveness in routine care. Our study may also be limited by dropouts and low recruitment, which could lead to bias in per-protocol analysis. Furthermore, our study was not blinded and analysed by intention-to-treat. The additional monthly nurse visits for the intervention group may have also introduced biases, which should have been taken into consideration and controlled. In order to minimise and compensate for these limitations, controlled randomisation was conducted. There is also uncertainty about the sustainability of the reduction in HbA1c levels and patient compliance when the sponsorship of test strips ceases.

In our study, SMBG practice resulted in improved glycaemic control, which could be due to increased empowerment among the patients in the intervention group, thus increasing the rate of glycaemic target 
achievement. However, routine use of SMBG may not be appropriate for patients with reasonably well-controlled type 2 DM. Further studies should be done to assess the role of SMBG and its cost-effectiveness in the management of patients with less well-controlled type 2 DM.

\section{REFERENCES}

1. Ministry of Health, Malaysia. The Third National Health Morbidity Survey (NHMS III) Diabetes Group. Malaysia: Ministry of Health, 2006.

2. Mastura I, Chew BH, Lee PY, et al. Control and Treatment Profiles of 70,889 Adult Type 2 Diabetes Mellitus Patients in Malaysia - A Cross Sectional Survey in 2009. Int J Collaborative Res Intern Med Public Health 2011; 3:98-113.

3. Mafauzy M Hussein Z Chan SP. The status of diabetes control in Malaysia: results of Diabcare 2008. Med J Malaysia 2011; 66:175-81.

4. Haliza AM, Roslan Johari MG, Badrulnizam M, et al. Management of Patients with Type II Diabetes Mellitus in $\mathrm{MOH}$ Hospitals and Health Centres. J Health Manage 2006; 4:73-87.

5. Intensive blood-glucose control with sulphonylureas or insulin compared with conventional treatment and risk of complication in patients with type 2 diabetes (UKPDS 33). UK Prospective Diabetes Study (UKPDS) Group. Lancet 1998; 352: 837-53. Erratum in: Lancet 1999; 354:602.

6. American Association of Clinical Endocrinologists. The American Association of Clinical Endocrinologists Medical Guidelines for the Management of Diabetes Mellitus: the AACE system of intensive diabetes self-management--2002 update. Endocr Pract 2002; 8 suppl 1:40-82.

7. Guerci B, Drouin P, Grange V, et al. Self-monitoring of blood glucose significantly improves metabolic control in patients with type 2 diabetes mellitus: the Auto-Surveillance Intervention Active (ASIA) study. Diabetes Metab 2003; 29:587-94.

8. Karter A, Ackerson LM, Darbinian JA, et al. Self-monitoring of blood glucose levels and glycemic control: the Northern California Kaiser Permanente Diabetes registry. Am J Med 2001; 111:1-9.

9. Charuruks N, Surasiengsunk S, Suwanwalaikorn S, et al. Impact of Self-monitoring of Blood Glucose in Diabetic Patients in Thailand. Point Care: J Near-Patient Test Technol 2006; 5:155-9.

10. Kibriya MG, Ali L, Banik NG, Khan AK. Home monitoring of blood glucose (HMBG) in Type-2 diabetes mellitus in a developing country. Diabetes Res Clin Pract 1999; 46:253-7.

11. Chidum E, Agbai D, Fidelis O, et al. Self-monitoring of blood glucose improved glycaemic control and 10-year coronary heart disease risk profile of type 2 diabetic patients. Chin Med J (Engl) 2011; 124:166-71.

12. Clinical Practice Guidelines Task Force. Clinical Practice Guidelines: Management of Type 2 Diabetes Mellitus. 4th ed. Malaysia: Ministry of Health, 2009.

13. Campbell MJ, Julious SA, Altman DG. Estimating sample sizes for binary, ordered categorical, and continuous outcomes in two group comparisons. BMJ 1995; 311:1145-8.

14. Welschen LM, Bloemendal E, Nijpels G, et al. Self-monitoring of blood glucose in patients with type 2 diabetes who are not using insulin: a systemic review. Diabetes Care 2005; 28:1510-7.

15. Fontbonne A, Billault B, Acosta $M$, et al. Is glucose self-monitoring beneficial in non-insulin- treated diabetic patients? Results of a randomized comparative trial. Diabetes Metab 1989; 15:255-60.
16. Muchmore DB, Springer J, Miller M. Self-monitoring of blood glucose in overweight type 2 diabetic patients. Acta Diabetol 1994; 31:215-9.

17. Allen BT, DeLong ER, Feussner JR. Impact of glucose self-monitoring on non-insulin-treated patients with type II diabetes mellitus. Randomized controlled trial comparing blood and urine testing. Diabetes Care 1990; 13:1044-50.

18. Schwedes U, Siebolds M, Mertes G; SMBG Study Group. Meal-related structured self-monitoring of blood glucose: effect on diabetes control in non-insulin-treated type 2 diabetic patients. Diabetes Care 2002; 25:1928-32.

19. Guerci B, Drouin P, Grangé V, et al. Self-monitoring of blood glucose significantly improves metabolic control in patients with type 2 diabetes mellitus: the Auto-Surveillance Intervention Active (ASIA) study. Diabetes Metab 2003; 29:587-94

20. Allemann S, Houriet C, Diem P, Stettler C. Self-monitoring of blood glucose in non-insulin treated patients with type 2 diabetes: a systematic review and meta-analysis. Curr Med Res Opin 2009; 25 2903-13.

21. McAndrew L, Schneider SH, Burns E, Leventhal E. Does patient blood glucose monitoring improve diabetes control? A systematic review of the literature. Diabetes Educ 2007; 33:991-1010.

22. McIntosh B, Yu C, Lal A, et al. Efficacy of self-monitoring of blood glucose in patients with type 2 diabetes mellitus managed without insulin: a systematic review and meta-analysis. Open Med 2010; 4:e102-13.

23. Mastura I, Mimi O, Piterman L, Teng CL, Wijesinha S. Self-monitoring of blood glucose among diabetes patients attending urban government health clinics. Med J Malaysia 2007; 62:147-51.

24. Tan MY, Magarey J. Self-care practices of Malaysian adults with diabetes and sub-optimal glycaemic control. Patient Educ Couns 2008; 72:252-67.

25. Adams AS, Mah C, Soumerai SB, et al. Barriers to self-monitoring of blood glucose among adults with diabetes in an HMO: a cross sectional study. BMC Health Serv Res 2003; 3:6

26. Clarke PM, Gray AM, Briggs A, et al. A model to estimate the lifetime health outcomes of patients with type 2 diabetes: the United Kingdom Prospective Diabetes Study (UKPDS) Outcomes Model (UKPDS no. 68). Diabetologia 2004; 47:1747-59.

27. Cameron C, Coyle D, Ur E, Klarenbach S. Cost-effectiveness of selfmonitoring of blood glucose in patients with type 2 diabetes mellitus managed without insulin. CMAJ 2010; 182:28-34.

28. Farmer AJ, Wade AN, French DP, et al. Blood glucose self-monitoring in type 2 diabetes: a randomised controlled trial. Health Technol Assess 2009; 13:iii-iv, ix-xi, 1-50.

29. Gallichan M. Self monitoring of glucose by people with diabetes: evidence based practice. BMJ 1997; 314:964-7.

30. Simon J, Gray A, Clarke P, et al. Cost effectiveness of self monitoring of blood glucose in patients with non-insulin treated type 2 diabetes: economic evaluation of data from the DiGEM trial. BMJ 2008; 336 : 1177-80.

31. O'Kane MJ, Bunting B, Copeland M, Coates VE; ESMON study group. Efficacy of self monitoring of blood glucose in patients with newly diagnosed type 2 diabetes (ESMON study): randomised controlled trial. BMJ 2008; 336:1174-7.

32. American Diabetes Association. Standard of Medical Care in Diabetes - 2010. Diabetes Care 2010; 33 suppl 1:S11-S61.

33. Zgibor JC, Simmons D. Barriers to blood glucose monitoring in a multiethnic community. Diabetes Care 2002; 25:1772-7. 